\section{Delayed resolution of pulmonary oedema after cocaine/heroin} abuse

\author{
Pietet G H M Raijmakers, A B Johan \\ Groeneveld, Marcel C M de Groot, \\ G J Jaap Teule, Lambertus G Thijs
}

\begin{abstract}
Pulmonary oedema lasting six days occurred in a 68 year old man after sniffing cocaine. He also had evidence of parenteral self-administration of heroin. Pulmonary microvascular filtration pressure and permeability were normal. Delayed resolution of the pulmonary oedema may have been caused by a cocaine-induced impairment of sodium and thus fluid transport across alveolar epithelium. Recognition may be important, since lowering filtration pressure with diuretics may not hasten resolution of oedema.
\end{abstract}

(Thorax 1994;49:1038-1040)

Parenteral drug abuse frequently results in acute respiratory failure and pulmonary oedema, associated with increased microvascular protein permeability caused by neurogenic factors, drugs, or contaminants in injectates. ${ }^{12}$ An overdose of inhaled cocaine can also lead to an acute lung injury presenting as acute alveolitis or permeability oedema, and this may be a major factor contributing to mortality. ${ }^{3-5}$ Pulmonary oedema may also be of hydrostatic origin because cocaine may elicit coronary vasoconstriction and insufficiency ${ }^{67}$ thereby raising left atrial pressure and fluid filtration in the lungs. We report a case of cocaine/heroin abuse and prolonged pulmonary oedema with normal pulmonary microvascular filtration pressure and permeability. It is postulated that delayed clearance of fluid across the alveolar membrane was responsible for the prolonged pulmonary oedema.

Department of Guclear Medile

Department of Internal Medicine, Free University Hospital, Boelelaan $1117,1081 \mathrm{HV}$ Amsterdam, The Netherlands

Reprint requests to: Dr P G H M Raijmakers. Received 30 September 1993

Returned to authors

I December 1993

Revised version received

23 December 1993

Accepted for publication

5 January 1994 present. Arterial blood gas analysis on ai pH $7 \cdot 24, \mathrm{PCO}_{2} 6 \cdot 8 \mathrm{kPa}, \mathrm{Po}_{2} 5 \cdot 3 \mathrm{kPa}$, reflecting arterial hypoxaemia and acute respiratory acidosis because of alveolar hypoventilation. A chest radiograph revealed bilateral infiltrates compatible with pulmonary oedema. Although the patient regained consciousness after an intravenous injection of $0.4 \mathrm{mg}$ of the opiate antagonist naloxone, he had to be intubated and artificially ventilated because of respiratory failure. There was no evidence of aspiration of gastric contents. Aspirated sputum was bloodstained during the first two days of admission.

In addition, a metabolite of cocaine (benzoylecgonine) and opiates were found in a urine sample obtained shortly after admission. The blood sugar level was normal $(8.5 \mathrm{mmol} / \mathrm{l})$. During the first three days white blood cell counts declined from 7.3 to $4.5 \times 10^{9} / 1$, with $1 \%$ eosinophils and $70 \%$ neutrophils. Blood and sputum cultures were negative. Bronchoalveolar lavage fluid samples obtained on the third day were negative for alveolar proteinosis, Pneumocystis carinii, and cytomegalovirus. Cell differentiation revealed a supranormal fraction of neutrophils (50\%) and lymphocytes $(20 \%)$ with a subnormal fraction of macrophages $(25 \%)$; there were $5 \%$ epithelial cells and no eosinophils.

In order to establish the cause of pulmonary oedema, electrocardiography and echocardiography were performed on the first day and there were no signs of myocardial ischaemia or dysfunction. A thermodilution balloon-tipped pulmonary artery catheter was inserted. Mean arterial pressure $(\mathrm{mm} \mathrm{Hg}$ ) was also measured (table). The plasma colloid osmotic pressure (PCOD) was calculated using a nomogram from measured total protein and albumin concentrations. The pulmonary microvascular hydrostatic pressure (PMv) is calculated as (mean pulmonary arterial - capillary wedge pressure) $\times 0.4+$ pulmonary capillary wedge pressure, so that the difference between PMv and PCOD is the estimated microvascular filtration pressure. Each time it was measured, pulmonary wedge pressure was $\leqslant 15 \mathrm{~mm} \mathrm{Hg}$ and the PCoD was also normal. ${ }^{2}$ The PMv - PCoD was negative, favouring resorption rather than filtration of fluid (table). Pulmonary microvascular permeability was assessed using a dual radionuclide method and scintillation detection probes positioned over the lungs, allowing the non-invasive determination of the ${ }^{67} \mathrm{Ga}$-labelled transferrin transport rate - that

Haemodynamic and pulmonary variables (mean daily values of 3-4 observations)

\begin{tabular}{|c|c|c|c|}
\hline & Day 1 & Day 2 & Day 3 \\
\hline HR (beats/min) & 102 & 94 & 86 \\
\hline $\mathrm{MAP}(\mathrm{mm} \mathrm{Hg})$ & 75 & 74 & 76 \\
\hline $\mathrm{CO}(1 / \mathrm{min})$ & $8 \cdot 0$ & $7 \cdot 0$ & $7 \cdot 0$ \\
\hline $\mathrm{CVP}(\mathrm{mm} \mathrm{Hg})$ & $6 \cdot 7$ & $8 \cdot 0$ & $7 \cdot 5$ \\
\hline MPAP (mm Hg) & 23 & 21 & 21 \\
\hline PCWP (mm Hg) & 10 & 10 & 11 \\
\hline PMV (mm Hg) & $15 \cdot 0$ & $14 \cdot 4$ & $15 \cdot 2$ \\
\hline PCOD (mm Hg) & $17 \cdot 4$ & $15 \cdot 2$ & $19 \cdot \overline{6}$ \\
\hline PLI $\left(\times 10^{-3} / \mathrm{min}\right)$ & 8.9 & 9.9 & $8 \cdot 2$ \\
\hline Arterial $\mathrm{PO}_{2}(\mathrm{kPa})$ & $10 \cdot 1$ & $10 \cdot 4$ & $11 \cdot 8$ \\
\hline $\mathrm{FIO}_{2}$ & 0.40 & 0.35 & 0.35 \\
\hline PEEP $\left(\mathrm{cm} \mathrm{H}_{2} \mathrm{O}\right)$ & 8 & 8 & 8 \\
\hline
\end{tabular}

$\mathrm{HR}=$ heart rate, $\mathrm{MAP}=$ mean arterial pressure, $\mathrm{CO}=$ cardiac output, $\mathrm{CVP}=$ central venous pressure, $\mathrm{MPAP}=$ mean pulmonary arterial pressure, $\mathrm{PCW}=$ pulmonary capillary wedge pressure, PMV =pulmonary micro PLI = pul pressure, PcOD plasma colloid osmotic pressure, PLI pulmonary leak index $\mathrm{FrO}_{2}=$ inspiratory oxygen concentration, PEEP = positive end expiratory pressure. PCOD and PLI were measured at 12, 36, and 60 hours after admission. 


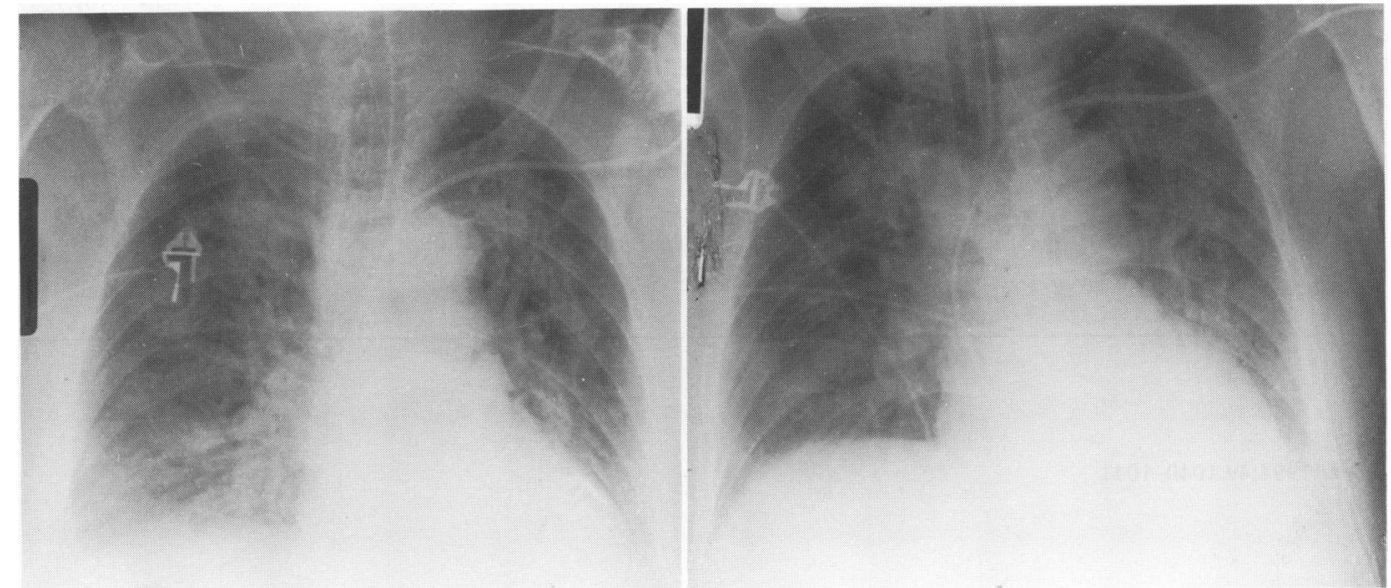

Chest radiographs taken 2-3 hours before measurement of pulmonary leak index on days 2 and 3 after admission showing bilateral pulmonary oedema.

is, the pulmonary leak index (PLI) - across pulmonary microvessels as a sensitive and specific measure of permeability. ${ }^{8}$ The normal PLI is $8.2(3 \cdot 7) \times 10^{-3} / \mathrm{min}$ (mean (SD) for 12 patients prior to elective vascular surgery). The PLI typically rises threefold in cases of increased permeability, even in the absence of overt alveolar oedema. ${ }^{8}$ Measured at 12,36 , and 60 hours after admission to the ICU, the PLI was normal (table) despite the fact that the chest radiograph, obtained 2-3 hours before each PLI measurement, showed persistent alveolar oedema.

Mechanical ventilation had to be continued for six days after admission because of persistent respiratory insufficiency and bilateral pulmonary oedema on the chest radiograph. Fluid balance, uncorrected for insensible losses, was positive $(2633 \mathrm{ml})$ on the first day, negative $(-186 \mathrm{ml})$ on the second day, and positive $(975 \mathrm{ml})$ on the third day. The patient received $40 \mathrm{mg}$ frusemide intravenously on the second and third day. On day 7 the chest radiograph showed a reduction of the pulmonary oedema. After extubation the patient admitted the use of intranasal cocaine, but denied intravenous use of opiates. Seventeen days after admission he left hospital.

\section{Discussion}

In our patient abuse of cocaine/heroin resulted in alveolar hypoventilation, pulmonary oedema, and respiratory failure. The diffuse infiltrates might have been caused by a hypersensitivity reaction to inhaled cocaine, the socalled "crack lung". 5 This is unlikely, however, because of the absence of fever, bronchospasm, and eosinophilia in the blood and bronchoalveolar lavage fluid, even in the presence of bloodstained sputum which, together with pulmonary haemosiderosis, is common in chronic cocaine smokers and sniffers. ${ }^{45}$ The clinical picture was therefore consistent with pulmonary oedema, a common complication of an overdose of drugs including cocaine..$^{1-46}$ Irrespective of its origin, alveolar oedema clears within 72 hours at most. ${ }^{9}$ Hence the persistence of oedema in our patient was caused by continued fluid filtration, delayed resolution, or both. The former was unlikely, however, since cardiac function, pulmonary hydrostatic, and plasma colloid osmotic pressures were normal. Moreover, microvascular permeability was normal. The PLI increases during inflammation as a result of increased protein permeability ${ }^{8}$ so that the normal PLI in our patient also argues against a hypersensitivity alveolitis. Increased fluid filtration was therefore not responsible for the oedema, although transient cocaineinduced or heroin-induced and neutrophilmediated damage of the microvasculature and increased permeability ${ }^{12}$ before haemodynamic and PLI measurements were performed may have been responsible for its development. The latter is supported by the relatively high number of neutrophils in the bronchoalveolar lavage fluid in the absence of bacterial infection.

Experimental and human studies indicate that clearance of alveolar fluid is, among other things, determined by active soaium transport across alveolar epithelium, that can be blocked by topical amiloride. ${ }^{9}$ Since cocaine, at clinically encountered blood concentrations, is a potent blocker of cardiac sodium channels, ${ }^{710}$ inhalation of the drug in our patient may have caused the prolonged pulmonary oedema through impairment of active sodium transport and thus fluid clearance across the alveolar wall. There was no evidence of cardiac toxicity - that is, severe tachycardia and slowing of intracardiac conduction - but this does not exclude blockade of sodium channels in the alveolar epithelium by inhaled cocaine. Cocaine may have impaired the fluid clearance for several days since metabolites, some of which block cardiac sodium channels similar to cocaine itself, are detectable in the urine for up to 48 hours after a single dose of the drug. ${ }^{710}$ Alternatively, a drug-induced, neutrophilmediated injury of epithelial cells might have hampered clearance of the alveolar oedema.

1 Duberstein JL, Kaufman DM. A clinical study of an epidemic of heroin intoxication and heroin-induced pulmonary edema. Am f Med 1971;51:704-14.

2 Sprung CL, Rackow EC, Fein IA, Jacob AI, Isikoff SK The spectrum of pulmonary edema: differentiation of cardiogenic, intermediate, and non-cardiogenic forms of pulmonary edema. Am Rev Respir Dis 1981;124:718.

3 Cucco RA, Yoo OH, Cregler L, Chang JC. Nonfatal pul mocco RA, Yoo OH, Cregler L, Chang JC. Nonfatal pulRespir Dis 1987;136:179-81. 
4 Murray RJ, Smialek JE, Golle M, Albin RJ. Pulmonary artery medial hypertrophy in cocaine users without foreig particle microembolization. Chest 1989;96:1050-3.

5 Forrester JM, Steele AW, Waldron JA, Parsons PE. Crack lung: an acute pulmonary syndrome with a spectrum of clinical and histopathological findings. Am Rev Respir Dis 1990;142:462-7.

6 Warner EA. Cocaine abuse. Ann Intern Med 1993;119:22635.

7 Kloner RA, Hale S. Unraveling the complex effects of cocaine on the heart. Circulation 1993;87:1046-7.
8 Raijmakers PGHM, Groeneveld ABJ, Schneider AJ, Teule GJJ, Van Lingen A, Eysman L, et al. Transvascular transport of ${ }^{67} \mathrm{Ga}$ in the lungs after cardiopulmonary bypass surgery. Chest 1993;104:1825-32.

9 Matthay MA, Wiener-Kronish JP. Intact epithelial barrier function is critical for the resolution of alveolar edema in humans. Am Rev Respir Dis 1990;142:1250-7.

10 Crumb WJ, Clarkson CW. Characterisation of the sodium channel blocking properties of the major metabolites of cocaine in single cardiac myocytes. 7 Pharmacol Exp Ther 1993;261:910-7.

\section{Eosinophilic endomyocardial disease due to high grade chest wall sarcoma}

\author{
A Hussain, P JE Brown, B C Thwaites, \\ A G Hastings
}

\begin{abstract}
Eosinophilic endomyocardial disease is characterised by persisting blood eosinophilia and acute endocardial lesions which progress to endomyocardial fibrosis. In most cases the cause is unknown but it has been described in association with malignant tumours. A fatal case is presented of a 64 year old woman with this disease due to a high grade sarcoma of the chest wall, an association not previously reported.
\end{abstract}

(Thorax 1994;49:1040-1041)

\section{Department of \\ Medicine \\ A Hussain \\ P J E Brown \\ B C Thwaites \\ Department of \\ Pathology \\ A G Hastings}

Ashington Hospital, Ashington NE63 0SA, UK

Reprint requests to: Dr A Hussain.

Received 23 June 1993 Returned to authors 25 August 1993

Revised version received

20 September 1993

Accepted for publication

6 January 1994

\section{Case report}

A 64 year old housewife presented with a two month history of general malaise, anorexia, effort dyspnoea causing breathlessness on minimal exertion, and a two week history of increasingly severe left upper posterior chest and shoulder pain. There was no history of haemoptysis or oedema. The patient was a current smoker of 40 pack-years with no relevant past medical history. Physical examination revealed evidence of recent weight loss, dyspnoea at rest with a respiratory rate of $24 /$ minute, without cyanosis, lymphadenopathy or finger clubbing. She was afebrile with a regular heart rate of $90 /$ minute, blood pressure $140 / 70 \mathrm{~mm} \mathrm{Hg}$, with a weight loss, non-productive cough, progressive third heart sound but no murmurs. Tenderness without palpable swelling was present over the left second and third ribs posteriorly. There was dullness to percussion at both bases with generalised expiratory wheezing on auscultation. Examination of the thyroid, abdomen and central nervous system showed no abnormality.

Laboratory investigations showed significant eosinophilia of $22 \times 10^{9} / 1$ of a total white count of $46.1 \times 10^{9} / \mathrm{l}$, haemoglobin $11.0 \mathrm{~g} / \mathrm{dl}$, ESR $50 \mathrm{~mm} /$ hour (Westergren). Serial chest radiographs with views of the left upper ribs showed a pleural-based opacity in the left upper zone peripherally associated with rapid bone destruction at the left second rib posteriorly and a pathological fracture of the left third rib. There were small bilateral pleural effusions present initially with subsequent consolidation of the right lower lobe after 10 days. The electrocardiogram showed sinus tachycardia with widespread $T$ wave inversion. Echocardiography showed undilated chambers with significant asymmetrical thickening of the posterior wall of the left ventricle. On apical views there was dramatic thickening of the lateral wall of the left ventricle with binding down of the posterior mitral valve leaflet. Mitral regurgitation was demonstrated. Urinalysis, serum levels of urea, creatinine, electrolytes, and liver function tests were all normal. A fine needle aspiration of the pleural-based mass showed numerous large malignant cells with many surrounding eosinophils.

The patient required narcotic analgesia for pain relief and commenced oral prednisolone $40 \mathrm{mg}$ daily resulting in rapid resolution of her eosinophilia, but she deteriorated progressively with dyspnoea due to heart failure which was unresponsive to treatment and died 21 days after admission.

Post mortem examination showed that the apex of the left upper lobe was tethered to thickened pleura by a $10 \mathrm{~cm}$ partially necrotic tumour mass that was eroding and partially destroying the second, third, and fourth ribs posteriorly. Histological examination of this tumour revealed a high grade sarcomatous neoplasm (figure) with moderate numbers of bizarre multinucleate giant cells. Epithelial markers were negative on immunohisto- 